University of South Carolina

Scholar Commons

$1-15-2007$

\title{
Radiation-Induced Current in Quantum Wires with Side-Coupled Nanorings
}

Yuriy V. Pershin Dr

University of South Carolina - Columbia, pershin@physics.sc.edu

Carlo Piermarocchi

Follow this and additional works at: https://scholarcommons.sc.edu/phys_facpub

Part of the Physics Commons

\section{Publication Info}

Published in Physical Review B, ed. Gene D. Sprouse, Volume 75, Issue 3, 2007, pages 035326-1-035326-6.

Pershin, Y. V., \& Piermarochhi, C. (2007). Radiation-induced current in quantum wires with side-coupled nanorings. Physcial Review B, 75(3), 035326-1 - 035326-6. DOI: 10.1103/PhysRevB.75.035326

(c) Physical Review B, 2007, American Physical Society

This Article is brought to you by the Physics and Astronomy, Department of at Scholar Commons. It has been accepted for inclusion in Faculty Publications by an authorized administrator of Scholar Commons. For more information, please contact digres@mailbox.sc.edu. 


\title{
Radiation-induced current in quantum wires with side-coupled nanorings
}

\author{
Yuriy V. Pershin* and Carlo Piermarocchi \\ Department of Physics and Astronomy, Michigan State University, East Lansing, Michigan 48824-2320, USA
}

(Received 26 October 2006; revised manuscript received 19 November 2006; published 17 January 2007)

\begin{abstract}
Photocurrent generation is studied in a system composed of a quantum wire with side-coupled quantum rings. The current generation results from the interplay of the particular geometry of the system and the use of circularly polarized radiation. We study the energy-momentum conservation for optical transitions involving electrons moving forward and backwards in the wire. Due to the lack of time-reversal symmetry in the radiation, the optical transitions depend on the direction of motion of the electrons, leading to a current at zero bias voltage. The photocurrent increases with the number of rings within a wide range of physical parameters. A weak nonlinear dependence of the current in the number of rings, related to quantum interference effects, is also predicted. This geometry suggests a scalable method for the generation of sizeable photocurrents based on nanoscale components.
\end{abstract}

DOI: 10.1103/PhysRevB.75.035326

PACS number(s): 73.21.Hb, 72.40.+w, 72.30.+q

\section{INTRODUCTION}

There is currently a significant theoretical and experimental interest in radiation-induced currents in confined electron systems. ${ }^{1-9}$ In particular, many investigations have focused on mechanisms for photocurrent generation based on nanoscale components. We have recently investigated a mechanism for photocurrent generation in a bent quantum wire by circularly polarized radiation. ${ }^{1}$ The curved geometry provides the optimal condition for exploiting the broken timereversal symmetry in the circularly polarized electromagnetic field. The mechanism of photocurrent generation in curved structures results from the interplay of circularly polarized radiation and the peculiar geometry, which yields an effective time-dependent sliding potential for the electrons in the bent region. ${ }^{2}$ In fact, the radiation induced sliding potential induces an asymmetry in the scattering coefficients for electrons moving forward and backwards and a net current appears at zero bias voltage. Radiation-induced transitions that reverse the sign of the electron velocity play a key role in the photocurrent generation. We found that for a realistic set of parameters the calculated photocurrent in bent quantum wires is relatively weak, of the order of $\sim 10 \mathrm{pA}$.

In the present paper, we consider photocurrent generation in a quantum wire with side-coupled quantum rings. This is a scalable structure with a mechanism of photocurrent generation similar to the one described above. Circularly polarized radiation creates a sliding potential in all the quantum rings coupled to the wire. This potential asymmetrically changes the scattering coefficients through the whole structure. The advantage over a single bent quantum wire is in the higher sensitivity to the radiation, which is related to the fact that the electron propagation involves trajectories with multiple passes through the ring regions. The path in which the electromagnetic field affects the dynamics is more extended, and the changes in the scattering are more significant. Moreover, since there are many rings coherently coupled to the electromagnetic field, we are interested in exploring the nonlinear features related to the coherent behavior of the electron in the many-ring structure.

Figure 1(a) shows the system under investigation. We consider a chain of $N$ one-dimensional rings, of radius $R$, in the $x$ - $y$ plane, side-coupled (from the same side) to a straight quantum wire in the $x$ direction. The distance between the centers of adjacent rings is $L$. A circularly polarized electromagnetic radiation propagates in $z$ direction, perpendicular to $x-y$ plane. The quantum wire is also connected to two electron reservoirs [denoted by $L$ (left) and $R$ (right)] at zero bias voltage. We are interested in the photocurrent generated in such a system. We can assume that the quantum wire and quantum rings are narrow in the transverse directions, so that our consideration is limited only to electrons within the lowest transverse subband. A radiation acting on the straight segments of the wire will not generate a current, no matter what polarization is used. We will therefore neglect the effect of the electromagnetic radiation on the straight segments of the nanostructure.

We calculate the radiation-induced current in the structure in the ballistic transport regime. In order to calculate the current, we solve a radiation-dependent scattering problem for the electrons in the structure using a transfer matrix approach. The total transfer matrix is calculated as a product of transfer matrices associated to many smaller segments in the structure. The transmission properties at the wire-ring junctions are modeled by a unitary matrix which depend on a parameter describing the strength of wire-ring coupling. The current is calculated as a function of different coupling pa-

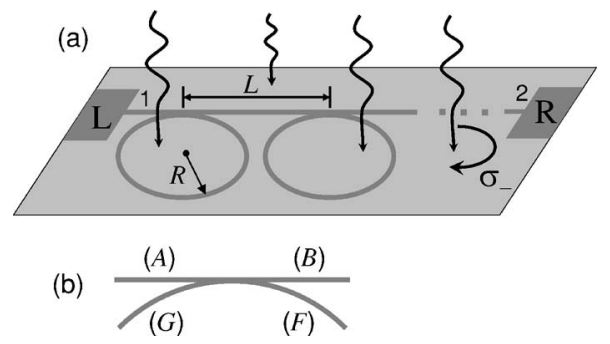

FIG. 1. (a) Quantum wire with $N$ side coupled quantum rings in the presence of circularly polarized radiation. The radiation breaks the symmetry for left- and right-moving electrons, inducing a photocurrent. (b) Definition of the wave function amplitudes near a single wire-ring junction. 
rameters and its scaling with the system size is considered. In a wide range of photon energies the photocurrent almost linearly increases with the number of quantum rings attached to the wire, but signatures of nonlinear effects are also observed. The paper is organized as follows. The mechanism of photocurrent generation is discussed in Sec. II, and the calculation scheme is introduced in Sec. III. In Sec. IV we present the results of calculations, and Sec. V contains concluding remarks.

\section{PHOTOCURRENT GENERATION MECHANISM}

We consider the interaction of electrons confined in a circular segment of the structure with circularly polarized electromagnetic radiation propagating perpendicularly to the quantum wire plane. The interaction term in the Hamiltonian can be written as ${ }^{1}$

$$
-\boldsymbol{d} \boldsymbol{E}=2 e R E_{0} \sin \left(\frac{s}{R} \pm \omega t\right),
$$

where $s$ is a coordinate along the ring, $\boldsymbol{d}=-e \boldsymbol{r}$ is the dipole moment operator. The electric field in the radiation is written as $\boldsymbol{E}=E_{0} \cos (\omega t) \hat{x} \pm E_{0} \sin (\omega t) \hat{y}$, where $E_{0}$ and $\omega$ are the electric field amplitude and frequency, $\hat{x}$ and $\hat{y}$ are unit vectors in the $x$ and $y$ directions (in the quantum wire plane), and \pm corresponds to a $\sigma_{ \pm}$circular polarization. According to Eq. (1), the electrons in the circular segment of the quantum wire (QW) are subjected to a potential that moves forwards or backwards depending on the helicity of the circularly polarized light. A similar sliding potential describes the interaction of electrons with a traveling acoustic wave. ${ }^{12}$ In our case, the effective wavelength of the traveling wave is determined by the quantum wire curvature and is $2 \pi R$.

The time-dependent potential in Eq. (1) induces transitions between different states. Figure 2(a) shows the transition scheme for an infinite quantum wire bent into a coil. In this coil geometry there is a full translational symmetry with respect to the coordinate $s$, leading to the exact energy and momentum conservation in the transition. This case must be discussed first in order to better understand the physical behavior of our structure, in which the translational symmetry is broken due to the presence of straight and curved segments. For $\sigma_{-}$radiation, the exact energy-momentum conservation conditions read

$$
\begin{gathered}
E\left(k^{\prime \prime}\right)=E\left(k^{\prime}\right) \pm \hbar \omega, \\
k^{\prime \prime}=k^{\prime} \pm \frac{1}{R} .
\end{gathered}
$$

Here $k^{\prime}$ and $k^{\prime \prime}$ are $k$-vectors of the initial and final electron states, \pm corresponds to a process of absorption and emission of a photon, and $1 / R$ is the $k$-vector of the sliding potential (1). In the case of $\sigma_{+}$radiation, \pm in Eq. (2) should be changed to $\mp$. We notice that this transition scheme is similar to the transition scheme in quantum ring illuminated by circularly polarized radiation. ${ }^{7}$

The key role in the current generation is played by transitions with $k^{\prime} k^{\prime \prime}<0$, i.e., when the direction of electron mo-
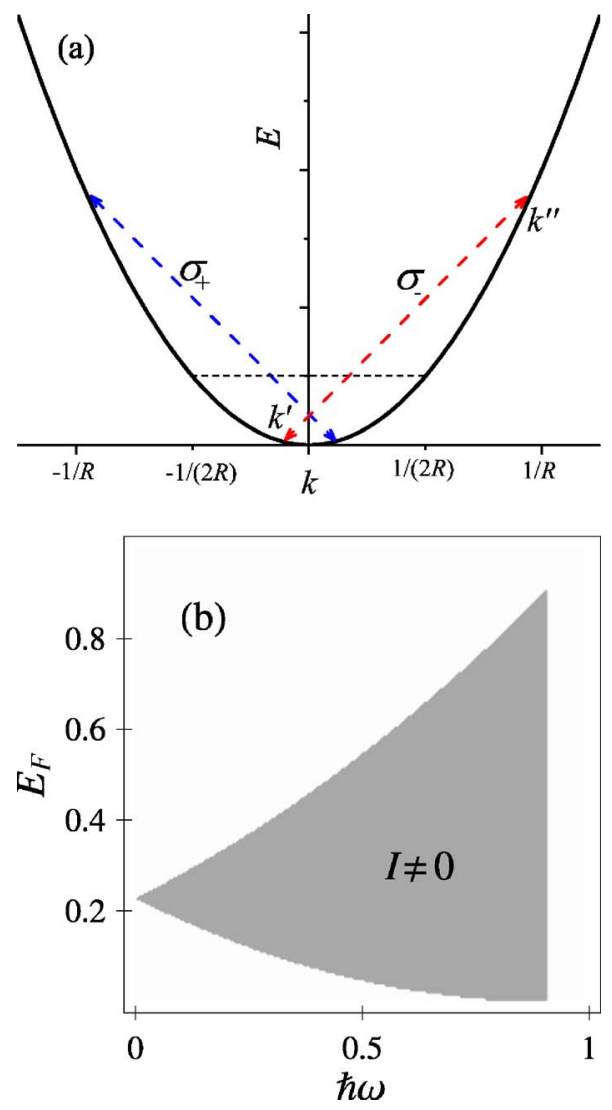

FIG. 2. (Color online) (a) Transitions induced by $\sigma_{+}$and $\sigma_{-}$ radiation. (b) Region of $\left(E_{F}, \hbar \omega\right)$ space with nonzero current. All calculations presented in this paper have been made using $m^{*}$ $=0.067 m_{e}, R=25 \mathrm{~nm}$, and $E_{0}=250 \mathrm{~V} / \mathrm{m} . E_{F}$ and $\hbar \omega$ are in $\mathrm{meV}$.

tion is reversed. Figure 2(a) gives examples of such transitions. Without the radiation, the electron currents due to the left- and right-moving electrons compensate each other. Radiation-induced transitions with $k^{\prime} k^{\prime \prime}<0$ correspond to a change in the backscattering of only one kind of electron (for example, the left moving). Therefore, the net current becomes nonzero at certain values of the Fermi energy $E_{F}$, namely, when the occupation probabilities for the states $k^{\prime}$ and $k^{\prime \prime}$ are different.

From Eqs. (2) and (3) we can find that, for a fixed value of the electromagnetic frequency, the initial and final momentum for the electron involved in the transition can be explicitly written as

$$
\begin{aligned}
& k^{\prime}=\frac{m R \omega}{\hbar}-\frac{1}{2 R}, \\
& k^{\prime \prime}=\frac{m R \omega}{\hbar}+\frac{1}{2 R} .
\end{aligned}
$$

It is clear that at zero temperature a current can be induced only when $\hbar^{2}\left(k^{\prime}\right)^{2} /\left(2 m R^{2}\right)<E_{F}<\hbar^{2}\left(k^{\prime \prime}\right)^{2} /\left(2 m R^{2}\right)$ and $k^{\prime}$ $<0$. This can be satisfied only for some values of the Fermi energy and photon energy. Figure 2(b) shows a region of the $\left(E_{F}, \hbar \omega\right)$ space where these conditions are fulfilled. 


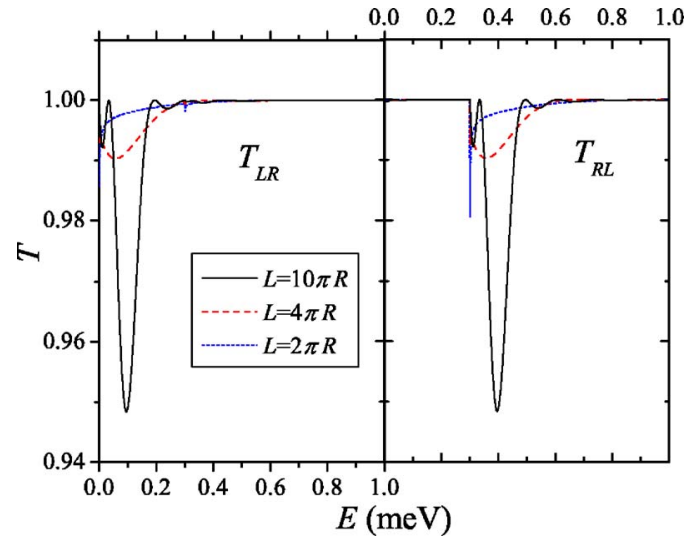

FIG. 3. (Color online) Total left-right and right-left transmission probabilities through curved quantum wires of different curved region lengths $L$ as indicated in the inset. The circularly polarized radiation introduces an asymmetry in the transmission probabilities. In the figure we considered $\hbar \omega=0.3 \mathrm{meV}$ and the geometrical potential was neglected.

This analysis applies to the case of a perfect infinite coilshaped wire. The energy-momentum conservation for a finite system, such as a curved quantum wire, ${ }^{1}$ is different than the one defined by Eqs. (2) and (3). In particular, the lack of the full translation symmetry implies that Eq. (3) is no longer valid. The numerical calculations presented in Fig. 3 refer to a single curved quantum wire, consisting of two straight segments smoothly connected by an arc of total length $L$. We plot there the total transmission probability for an electron of energy $E$ from the left to the right $\left(T_{L R}\right)$ and from the right to the left $\left(T_{R L}\right)$ in the presence of a $\sigma_{-}$polarized radiation. The definition of such probabilities is given below in Eq. (8), and the numerical calculation is the same as that discussed in Ref. 1. The figure shows how the shape of the transmission coefficient is modified by the finite size of the ring. Instead of a peak at a particular energy determined by the energy momentum conservation, a broader shape for the transmission coefficient appears. Notice that the transmission probabilities $T_{R L}$ and $T_{L R}$ for the $L=10 \pi R$ quantum wire show clear peaks exactly at the energies defined by Eqs. (2) and (3). The peak in $T_{L R}$ corresponds to a transition with absorption of a photon $\left[k^{\prime} \rightarrow k^{\prime \prime}\right.$ transition in Fig. 2(a)], the peak in $T_{R L}$ is related to emission of a photon ( $k^{\prime \prime} \rightarrow k^{\prime}$ transition). By decreasing $L$ these peaks shift to lower energy and become broader. For $L=2 \pi R$, only small peaks are observed. In this short ring limit, momentum conservation is released and only the energy conservation condition is maintained (i.e., electrons with $E<\hbar \omega$ cannot emit $\hbar \omega$ photons). Moreover, the shape of the transmission coefficients in this case follow the typical $1 / \sqrt{E}$ dependence of the one-dimensional density of states.

\section{CALCULATION METHOD}

In the ballistic transport regime, the electric current from the left $(L)$ to the right $(R)$ reservoirs can be written using a generalization $^{10-12}$ of the Landauer-Büttiker formula ${ }^{13}$ that takes into account the presence of a radiation field of frequency $\omega / 2 \pi$,

$$
I=\frac{2 e}{h} \sum_{n} \int_{0}^{\infty}\left[T_{R, L}(E+n \hbar \omega, E) f_{\mu_{L}}-T_{L, R}(E+n \hbar \omega, E) f_{\mu_{R}}\right] d E
$$

Here $e$ is the electron charge and $T_{R, L}(E+n \hbar \omega, E)$ is the probability that an electron of energy $E$ in the left reservoir is transmitted to the right reservoir in a state of energy $E$ $+n \hbar \omega$. Since we are going to study the current in the absence of external bias, i.e., at $\mu_{L}=\mu_{R}=\mu$, Eq. (6) can be rewritten as

$$
I=\frac{2 e}{h} \int_{0}^{\infty} \Delta T(E) f_{\mu} d E
$$

where

$$
\begin{aligned}
\Delta T(E) & =\sum_{n}\left[T_{R, L}(E+n \hbar \omega, E)-T_{L, R}(E+n \hbar \omega, E)\right] \\
& =T_{R L}(E)-T_{L R}(E) .
\end{aligned}
$$

We will calculate the photocurrent taking into account only single photon absorption and emission processes. This is equivalent to restrict $n$ to $-1,0,1$ values. The wave function in the first region [near the left contact, see Fig. 1(a)] can then be written as

$$
\psi_{1}=\sum_{n=-1,0,1}\left(A_{n,+} e^{i k_{n} x}+A_{n,-} e^{-i k_{n} x}\right) e^{-[i(E+n \hbar \omega) t] / \hbar}
$$

where $k_{n}=\sqrt{2 m^{*}(E+n \hbar \omega) / \hbar^{2}}$. The wave function $\psi_{2}$ near the right contact is given by the same expression with $A$ replaced by $B$. We use the transfer matrix method ${ }^{16}$ to calculate the transmission through the whole system. The total transfer matrix $M$ couples a vector $(A)$ $=\left(A_{-1,+}, A_{-1,-}, A_{0,+}, A_{0,-}, A_{1,+}, A_{1,-}\right)^{T}$ to a vector $\quad(B)$ $=\left(B_{-1,+}, B_{-1,-}, B_{0,+}, B_{0,-}, B_{1,+}, B_{1,-}\right)^{T}$ as

$$
(A)=M(B) .
$$

For a system of $N$ rings $M$ can be written as

$$
M=\mathcal{M}_{0}\left(\prod_{p=1}^{N-1} \mathcal{N}_{p} \mathcal{M}_{p}\right) \mathcal{N}_{p},
$$

where $\mathcal{M}_{p}$ is a transfer matrix of a straight quantum wire segment $p$ and $\mathcal{N}_{p}$ is a transfer matrix of the junction of the quantum wire with the $p$ th ring. We define the segment 0 as the part of quantum wire going from the $L$ reservoir to the first ring, the segment 1 is from the first ring to the second ring, and so on. The matrix $\mathcal{M}_{p}$ has a simple diagonal form 


$$
\left(\begin{array}{cccccc}
e^{-i k_{-1} L_{p}} & 0 & 0 & 0 & 0 & 0 \\
0 & e^{i k_{-1} L_{p}} & 0 & 0 & 0 & 0 \\
0 & 0 & e^{-i k_{0} L_{p}} & 0 & 0 & 0 \\
0 & 0 & 0 & e^{i k_{0} L_{p}} & 0 & 0 \\
0 & 0 & 0 & 0 & e^{-i k_{1} L_{p}} & 0 \\
0 & 0 & 0 & 0 & 0 & e^{i k_{1} L_{p}}
\end{array}\right)
$$

where $L_{p}$ is the length of $p$ th segment.

In order to find the transfer matrix $\mathcal{N}_{p}$ we consider a single quantum wire-ring junction. The transfer matrix is obtained from the equations that couple the electron wave function in different branches of the junction. We formulate these equations using the Shapiro scheme, ${ }^{14,15}$ which requires that the scattering matrix $S$, connecting the incoming and outgoing waves at the junction, is unitary. Using the wave function amplitudes in the wire (A) defined in Eq. (9), and the similarly defined amplitudes in the ring near the junction in the regions $(B),(F)$, and $(G)$ [see Fig. 1(b)] we couple the ingoing and out-going waves in the junction as

$$
\left(\begin{array}{c}
A_{i,+} \\
B_{i,-} \\
F_{i,-} \\
G_{i,+}
\end{array}\right)=S\left(\begin{array}{c}
A_{i,-} \\
B_{i,+} \\
F_{i,+} \\
G_{i,-}
\end{array}\right)
$$

where $i=-1,0,1$ and the $S$ matrix is chosen in the form

$$
S=\left(\begin{array}{cccc}
0 & \sqrt{1-a^{2}} & a & 0 \\
\sqrt{1-a^{2}} & 0 & 0 & a \\
a & 0 & 0 & -\sqrt{1-a^{2}} \\
0 & a & -\sqrt{1-a^{2}} & 0
\end{array}\right) .
$$

Here, the parameter $0<a<1$ describes the splitting of an in-going wave between different out-going channels. The $a$ $\sim 0$ limit corresponds to a quantum ring detached from the wire, while the $a \sim 1$ corresponds to a wire with a loop. The actual value of $a$ depends on the microscopical structure of the junction. We will consider $a$ as a free parameter in our theory.

The next step is to match at the boundaries the wave function amplitudes $(F)$ and $(G)$ with the solution of the Schrödinger equation in the ring. The procedure has beed described in detail in Ref. 1. Here, we summarize the main steps of the derivation. Let us consider first the electron wave function in the ring $\psi_{r}$. The single electron Hamiltonian in the effective-mass approximation is given by

$$
H=-\frac{\hbar^{2}}{2 m^{*}} \frac{\partial^{2}}{\partial s^{2}}-\boldsymbol{d} \boldsymbol{E}
$$

where $m^{*}$ is the effective mass, $s$ is a coordinate along the ring. The dipolar interaction term $\boldsymbol{d}=-e \boldsymbol{r}$ has been introduced earlier in Eq. (1). We neglect the geometrical potential $U_{g}$ $=\hbar^{2} /\left(8 m^{*} R^{2}\right),{ }^{17}$ which is not expected to affect the total current in the system. Taking into account only single photon absorption and emission processes, corresponding to $n=-1,0,1$ in Eq. (8), we can write the electronic wave function in the form

$$
\psi_{r}(s, t)=\sum_{n=-1}^{1} f_{n}(s) e^{-[i(E+n \hbar \omega) t] / \hbar} .
$$

We consider the case of $\sigma_{-}$polarization, as shown in Fig. 1. The $\sigma_{+}$case is analogous. By substituting Eq. (16) in the time-dependent Schrödinger equation and neglecting multiphoton absorption and emission terms, we obtain

$$
\begin{gathered}
(E-\hbar \omega) f_{-1}+\frac{\hbar^{2}}{2 m^{*}} f_{-1}^{\prime \prime}=i e E_{0} R e^{-i(s / R)} f_{0}, \\
E f_{0}+\frac{\hbar^{2}}{2 m^{*}} f_{0}^{\prime \prime}=i e E_{0} R\left(e^{-i(s / R)} f_{1}-e^{i(s / R)} f_{-1}\right), \\
(E+\hbar \omega) f_{1}+\frac{\hbar^{2}}{2 m^{*}} f_{1}^{\prime \prime}=-i e E_{0} R e^{i(s / R)} f_{0} .
\end{gathered}
$$

By seeking for solutions of Eqs. (17)-(19) in the form $f_{-1}$ $=C_{-1} e^{i[\tilde{k}-(1 / R)] s}, f_{0}=C_{0} e^{i \tilde{k} s}$, and $f_{1}=C_{1} e^{i[\tilde{k}+(1 / R)] s}$, we obtain the following system of equations for the coefficients $C_{i}$ :

$$
\begin{gathered}
(E-\hbar \omega) C_{-1}-\frac{\hbar^{2}\left(\tilde{k}-\frac{1}{R}\right)^{2}}{2 m^{*}} C_{-1}-i e E_{0} R C_{0}=0, \\
(E) C_{0}-\frac{\hbar^{2} \widetilde{k}^{2}}{2 m^{*}} C_{0}+i e E_{0} R\left(C_{-1}-C_{1}\right)=0,
\end{gathered}
$$

$$
(E+\hbar \omega) C_{1}-\frac{\hbar^{2}\left(\tilde{k}+\frac{1}{R}\right)^{2}}{2 m^{*}} C_{1}+i e E_{0} R C_{0}=0 .
$$

By imposing that the matrix in the linear system of Eqs. (20)-(22) has determinant equal to zero, we obtain six complex values of $\tilde{k}$. The wave function in the ring can then be written as

$$
\psi_{r}(s, t)=\sum_{j=1}^{6} \sum_{n=-1}^{1} C_{n, j} e^{i\left[\tilde{k}_{j}+n(1 / R)\right] s} e^{-[i(E+n \hbar \omega) t] / \hbar} .
$$

Using the continuity of the wave function and the current conservation condition, we match, in the vicinity of the junction, the plane waves defined by $(G)$ and $(F)$ to $\psi_{r}$ at $s=0$ and $s=2 \pi R$, correspondingly. From the matching conditions we obtain

$$
F_{n, \pm}=\frac{1}{2} \sum_{j}\left(1 \pm \frac{\tilde{k}_{j}+\frac{n}{R}}{k_{n}}\right) C_{n, j}
$$




$$
G_{n, \pm}=\frac{1}{2} \sum_{j}\left(1 \pm \frac{\tilde{k}_{j}+\frac{n}{R}}{k_{n}}\right) e^{i\left[\tilde{k}_{j}+n(1 / R)\right] 2 \pi R} C_{n, j} .
$$

Moreover, from Eqs. (20) and (22) we can express $C_{ \pm 1, j}$ as a function of $C_{0, j}$. Therefore, we obtain 12 linear equations given by (13) involving 18 variables $A_{i, \pm}, B_{i, \pm}$, and $C_{0, j}$. We can write these equations as

$$
\mathcal{K}_{p}\left(\begin{array}{c}
A_{-1,+} \\
A_{-1,-} \\
A_{0,+} \\
A_{0,-} \\
A_{1,+} \\
A_{1,-} \\
C_{0,1} \\
C_{0,2} \\
C_{0,3} \\
C_{0,4} \\
C_{0,5} \\
C_{0,6}
\end{array}\right)=\left(\begin{array}{c}
\sqrt{1-a^{2}} B_{-1,+} \\
\sqrt{1-a^{2}} B_{0,+} \\
\sqrt{1-a^{2}} B_{1,+} \\
B_{-1,-} \\
B_{0,-} \\
B_{1,-} \\
0 \\
0 \\
0 \\
a B_{-1,+} \\
a B_{0,+} \\
a B_{1,+}
\end{array}\right),
$$

where $\mathcal{K}_{p}$ is a matrix. Multiplying Eq. (26) by $\mathcal{K}_{p}^{-1}$ we can find an expression for $A_{n, \pm}$ as a function of $B_{n, \pm}$. The coefficients in these expressions give the transfer matrix $\mathcal{N}_{p}$ defined in Eq. (11).

The total transfer matrix $\mathcal{M}$ is calculated using Eq. (11). The scattering coefficients for an electron coming, let us say, from the left contact are found from Eq. (10) by setting $(A)=\left(0, A_{-1,-}, 1, A_{0,-}, 0, A_{1,-}\right)^{T}$ and $(B)$ $=\left(B_{-1,+}, 0, B_{0,+}, 0, B_{1,+}, 0\right)^{T}$. The total transmission coefficient from the left to the right reservoir at the energy $E$ is then given by

$$
\begin{aligned}
\sum_{n=-1}^{1} T_{R, L}(E+n \hbar \omega, E)= & \left|B_{0,+}\right|^{2}+\frac{k_{1}}{k_{0}}\left|B_{1,+}\right|^{2} \\
& +\theta(E-\hbar \omega) \frac{k_{-1}}{k_{0}}\left|B_{-1,+}\right|^{2},
\end{aligned}
$$

where $\theta(z)$ is the step function. The total transmission from the right to left reservoir is determined in a similar way.

\section{RESULTS}

Using the above scheme we have calculated the photocurrent as a function of different parameters. Figure 4 shows the photocurrent as a function of photon energy for different values of the coupling parameter $a$ in a structure with a single ring. In the strong coupling limit $(a \sim 1)$ the electron trajectory involves mainly a single pass in the ring. In fact, according to Eq. (14), an electron is injected with a probability $a^{2}$ from one part of quantum wire into the ring and, after traveling within the ring, it proceeds forward into the quantum wire with the same probability. Therefore, in this limit, the photocurrent dependence on the photon energy is similar

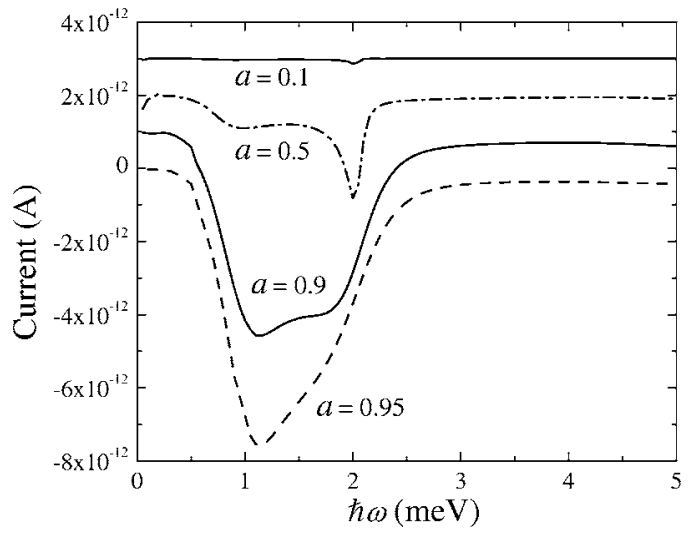

FIG. 4. Photocurrent in the system with a single ring as a function of photon energy for different values of the coupling constant $a$. For all plots, $E_{0}=250 \mathrm{~V} / \mathrm{m}, R=25 \mathrm{~nm}, \mu=1 \mathrm{meV}, T=1 \mathrm{~K}, m^{*}$ $=0.067 \mathrm{~m}_{e}$, and $L=100 \mathrm{~nm}$. All the curves other than $a=0.95$ have been vertically shifted by steps of $10^{-12}$ A for clarity.

to the one obtained in a bent quantum wire. ${ }^{1}$ In particular, we observe that the curve $a=0.95$ is characterized by a dominant peak in the vicinity of $E_{F}$.

The presence of this peak at the Fermi energy can be interpreted by considering the transmission probabilities for left- and right-moving electrons shown in Fig. 3. The total current is proportional to $T_{L R}(E)-T_{R L}(E)$ integrated up to the Fermi energy. It is clear from Fig. 3 that this integral would be maximal if $E_{F}$ is slightly smaller than $\hbar \omega$ since at this energy only the absorption processes in $T_{L R}$ contribute to the total current, and the reverse current due to $T_{R L}$ is zero. When the Fermi energy is bigger than the photon energy there is a contribution from $T_{R L}$ due to the emission of photons that compensate the left to right current. This leads to a decrease in the current, which eventually reaches zero when all emission and absorption processes balance $T_{L R}$ and $T_{R L}$.

The photocurrent behavior at smaller values of $a$ is more complex. Notice that starting at about $a=0.9$ a second peak emerges in the vicinity of $2 E_{F}$. At $a=0.5$ the peak at $2 E_{F}$ dominates becomes dominant. For smaller $a$ ( $a=0.1$ curve) the peak at $E_{F}$ has practically disappeared. This second peak

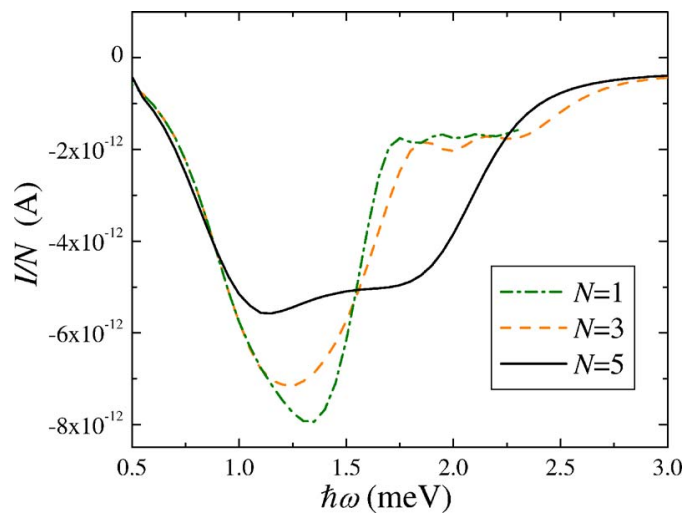

FIG. 5. (Color online) Normalized photocurrent $I / N$ as a function of the photon energy for different number of rings $(N)$ in the system. The coupling coefficient is $a=0.9$. Notice the strong dependence of the photocurrent profile on the number of rings. 


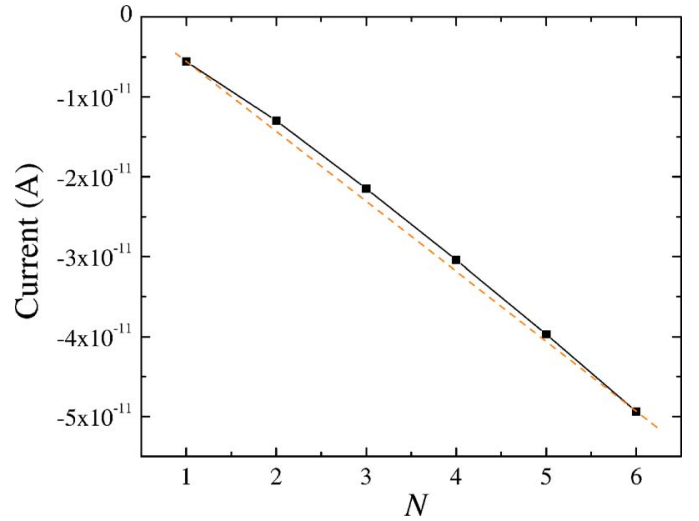

FIG. 6. (Color online) Peak value of the photocurrent $I_{\text {peak }}$ as a function of the number of rings $N$ calculated at $a=0.9$. For each value of $N$, the peak value of photocurrent $I_{\text {peak }}$ is determined as a minimum of $I(\hbar \omega)$. The dashed line connecting the first and last points is a guide for the eye. It helps to notice that the dependence $I_{\max }(N)$ slightly deviates from a linear dependence.

can be related to the interference between the two paths of the electron from $(A)$ to $(B)$ : a direct path and a path involving a single or multiple loops in the ring. Notice also that, as expected, the total photocurrent decreases for smaller $a$.

Figures 5 and 6 show the main results of the paper. These figures refer to a strong ring-wire coupling of $a=0.9$, which can give a strong current. We plot in Fig. 5 the photocurrent normalized to the number of rings $I / N$ as a function of photon energy for different number of rings in the system. Notice that photocurrent profile is changing as a function of $N$, which implies strong nonlinear effects. The normalized amplitude of the peak in the vicinity of $E_{F}$ increases and shifts to higher energies with $N$, while the peak in the vicinity of $2 E_{F}$ decreases. At low photon energies $\hbar \omega \lesssim 0.7 \mathrm{meV}$ the photocurrent scales linearly with $N$.

Notice that the peak value of the photocurrent $I_{\text {peak }}$ is almost a linear function of $N$ in the range investigated (the increase of the normalized amplitude in Fig. 5 is related to a positive offset). However, there are appreciable deviations from a pure linear dependence (see the dashed curve in Fig. 6). In fact, a fit to the numerical results give a quadratic dependence, which suggests that multiring interference affects the total current in the system.

Our calculations assume the ballistic transport regime. The ballistic approximation remains valid as long as the phase coherence length $l_{c}$ is longer than the electron path through the system. In state-of-the-art ballistic structures the phase coherence length could reach several microns. By estimating the electron path through a single unit of the structure as $L+2 \pi R$, we find that for $L=100 \mathrm{~nm}, R=25 \mathrm{~nm}$, and $l_{c}=2 \mu \mathrm{m}$ the transport through the total structure remains ballistic in systems with a number of rings $N \lesssim 6$. However, we believe that the discussed mechanism of current generation in a quantum wire with side-coupling rings is not limited to ballistic structures. In fact, recent calculations for a different geometry suggests the possibility of similar current generation mechanism in the diffusive regime. ${ }^{2}$

\section{CONCLUSIONS}

We have discussed photocurrent generation in a quantum wire-ring mesoscopic system by circularly polarized radiation. In contrast to previous schemes, the present system can be scaled to include a large number of rings, which may lead to stronger photocurrent. We have shown that the photocurrent increases almost linearly with the number of rings within a wide range of parameters. Moreover, we have observed a weak nonlinear dependence. From an experimental point of view, a mesoscopic system with a quantum wire and side-coupled rings can be fabricated using standard growth methods. This method of current generation could be explored also in carbon nanotube loops, in which transport properties have been recently experimentally investigated. ${ }^{18}$

\section{ACKNOWLEDGMENTS}

The authors are grateful to M. Dykman for many fruitful discussions. This research was supported by the National Science Foundation, Grant No. NSF DMR-0605801.
*Electronic address: pershin@ @ pa.msu.edu

${ }^{1}$ Yu. V. Pershin and C. Piermarocchi, Phys. Rev. B 72, 195340 (2005).

${ }^{2}$ L. I. Magarill and M. V. Éntin, JETP Lett. 78, 213 (2003); L. I. Magarill and A. V. Chaplik, Pis'ma Zh. Eksp. Teor. Fiz. 70, 607 (1999) [JETP Lett. 70, 615 (1999)].

${ }^{3}$ A. Fedorov, Yu. V. Pershin, and C. Piermarocchi, Phys. Rev. B 72, 245327 (2005); Yu. V. Pershin and C. Piermarocchi, Appl. Phys. Lett. 86, 212107 (2005).

${ }^{4}$ Y. Levinson and P. Wölfle, Phys. Rev. Lett. 83, 1399 (1999).

${ }^{5}$ C. Niu and D. L. Lin, Phys. Rev. B 62, 4578 (2000).

${ }^{6}$ S. Blom and L. Y. Gorelik, Phys. Rev. B 64, 045320 (2001).

${ }^{7}$ Yu. V. Pershin and C. Piermarocchi, Phys. Rev. B 72, 245331 (2005); 72, 125348 (2005).

${ }^{8}$ R. G. Mani, J. H. Smet, K. von Klitzing, V. Narayanamurti, W. B.
Johnson, and V. Umansky, Nature (London) 420, 646 (2002).

${ }^{9}$ M. A. Zudov, R. R. Du, L. N. Pfeiffer, and K. W. West, Phys. Rev. Lett. 90, 046807 (2003).

${ }^{10}$ S. Datta and M. P. Anantram, Phys. Rev. B 45, R13761 (1992).

${ }^{11}$ F. A. Maaø and L. Y. Gorelik, Phys. Rev. B 53, 15885 (1996).

${ }^{12}$ F. A. Maaø and Y. Galperin, Phys. Rev. B 56, 4028 (1997).

${ }^{13}$ R. Landauer, IBM J. Res. Dev. 1, 223 (1957); M. Buttiker, Phys. Rev. Lett. 57, 1761 (1986).

${ }^{14}$ B. Shapiro, Phys. Rev. Lett. 50, 747 (1983).

${ }^{15}$ M. Büttiker, Y. Imry, and M. Ya. Azbel, Phys. Rev. A 30, 1982 (1984).

${ }^{16}$ X. F. Wang and P. Vasilopoulos, Phys. Rev. B 72, 165336 (2005).

${ }^{17}$ R. C. T. da Costa, Phys. Rev. A 23, 1982 (1981).

${ }^{18}$ G. Refael, J. Heo, and M. Bockrath, cond-mat/0608365 (to be published). 